\title{
Metastatic Signet Ring Gastric Carcinoma Bypassing Virchow's node: An Unexpected Etiology of a Painful Neck Mass.
}

\author{
Arianna Di Stadio ${ }^{1}$, Luca D'Ascanio ${ }^{2}$, Gino Latini² ${ }^{2}$, Paolo Gradoni², Manlio Pandolfini ${ }^{2}$, \\ Tiziana Tamburrano ${ }^{2}$, Giampietro Ricci ${ }^{3}$, Massimo Maranzano ${ }^{4}$, and Michael Brenner ${ }^{5}$ \\ ${ }^{1}$ University of Perugia School of Medicine and Surgery \\ ${ }^{2}$ Ospedali Riuniti Marche Nord Presidio ospedaliero Santa Croce \\ ${ }^{3}$ University of Perugia Department of Medicine \\ ${ }^{4}$ Manchester University NHS Foundation Trust \\ ${ }^{5}$ University of Michigan
}

September 21, 2020

\begin{abstract}
Neck masses can be a challenge because several cancers metastasize in this area. Head and neck tumors are more commonly responsible of lymph nodes in neck. However, also gastric cancer can be metastatic in this area. We present a rare case of neck gastric meta with negative Virchow's lymph node.
\end{abstract}

Metastatic Signet Ring Gastric Carcinoma Bypassing Virchow's node: An Unexpected Etiology of a Painful Neck Mass.

Arianna Di Stadio, MD, $\mathrm{PhD}^{3^{*}}$, Luca D'Ascanio $\mathrm{MD}^{1^{*}}$, Gino Latini $\mathrm{MD}^{1}$, Paolo Gradoni $\mathrm{MD}^{1}$, Manlio Pandolfini $\mathrm{MD}^{1}$,

Tiziana Tamburrano $\mathrm{MD}^{2}$, Giampietro Ricci $\mathrm{MD}^{3}$, Massimo Maranzano ${ }^{4}$ and Michael J Brenner $\mathrm{MD}^{5}$

1: Department of Head and Neck Surgery, Institute of Otorhinolaryngology, Santa Croce Hospital AORMN, Fano, Italy

2: Institute of Oncology, Santa Croce Hospital AORMN, Fano, Italy

3: Department of Otolaryngology, University of Perugia, Perugia, Italy

4: CFU of Head and Neck Surgery, Division of Oral and Maxillofacial and Facial Plastic Surgery

Manchester University Foundation Trust

5: Department of Otolaryngology-Head and Neck Surgery, University of Michigan, Ann Arbor, MI, USA

*: equal contribution

Corresponding author: Dr. Arianna Di Stadio, Otolaryngology Department, Piazza Menghini 1, 06129 Perugia, Italy. E-mail: ariannadistadio@hotmail.com; arianna.distadio@unipg.it

Running title: Neck Metastasis with Unknown Primary 


\begin{abstract}
Neck masses can be a challenge because several cancers metastasize in this area. Head and neck tumors are more commonly responsible of lymph nodes in neck. However, also gastric cancer can be metastatic in this area. We present a rare case of neck gastric meta with negative Virchow's lymph node.
\end{abstract}

KEYWORDS: Neck mass, neck pain, lymph node, Virchow's lymph node, gastric cancer, head cancer, differential diagnosis.

KEY CLINICAL MESSAGE: Neck lymph nodes are common metastases of head and neck tumors. Other tumors, i.e gastric one, metastasize firstly in the clavicular fossa and then in the neck. Our case describes a rare gastric tumor with neck meta and negative Virchow's lymph node.

\title{
INTRODUCTION
}

Despite clinical practice guidelines to guide evaluation and management of neck masses [1], the diverse etiologies of neck masses may make accurate diagnosis challenging [2]. A neck mass in an adult most often represents lymphatic transit of metastasis from a mucosal squamous cell carcinoma or less commonly a primary tumor. Visceral malignancy [3] may also affect the head and neck, usually via lymphatic spread that classically presents as an enlarged Virchow's lymph node (VN), a left-sided supraclavicular fossa lymph node. Muscle metastasis may also occur via hematogenous spread [4,5]. We describe a rare case of signet ring cell-variant gastric adenocarcinoma that bypassed Virchow's node to involve the posterolateral neck.

\section{CASE PRESENTATION}

A 78-year-old white woman presented with a 6-month history of progressive left neck swelling, and she had recently developed pain on lateral neck movement. She had no other symptoms, and her medical history was negative history except for a clear cell renal cell carcinoma removed in May 2014 and without recurrence. Exam demonstrated a firm, hypomobile mass of the Level $\mathrm{V}$ neck, without any palpable masses in the clavicular fossa or other regions of the neck. Flexible endoscopy revealed no evident mucosal lesions of the upper aerodigestive tract, and ultrasound-guided biopsy was non-diagnostic.

Because of her cancer history, neck magnetic resonance imaging (MRI) with gadolinium, neck and thorax contrast computed tomography (CT), and total body Positron Emission Tomography (PET) CT were performed. Head and neck MRI (Figure 1AB ) and CT (Figure 1CD ) imaging demonstrated an enhancing posterolateral neck lesion measuring $37 \times 35 \times 15 \mathrm{~mm}$ mass adjacent to and invading the sternocleidomastoid muscle. Thoracic imaging did not show presence of masses or lymph nodes. \sout. Repeat fine needle aspirates performed under ultrasound guidance were non-diagnostic; the patient wished surgical removal and was therefore taken to surgery for endoscopy of the upper respiratory tract, excisional biopsy of the mass, and plan for formal neck dissection if squamous cell carcinoma was identified. Endoscopy showed no abnormality, and removal of the neck mass was performed with surrounding fibro-adipose tissue and sternocleidomastoid muscle.

Pathology of frozen section demonstrated cellular atypia consistent with gastric carcinoma (Figure 2 ABC) . Subsequent gastric endoscopy (performed under general anesthesia) identified a gastric ulcer located in the larger curvature of the stomach/anterior wall of pre-pyloric area. Several biopsies were performed in the stomach close and far from the identified lesion. The histologic analysis of the finding confirmed the diagnosis of signet-ring cell carcinoma pathology, HER2 negative.

Following multidisciplinary review, the patient started chemotherapy with FOLFOX. There was tumor progression despite therapy, and the regimen was therefore changed to FOLFIRI, with tumor regression at 10-months. The patient was still in remission at the last follow-up in August 2020.

\section{DISCUSSION}

This case underscores importance of definitive diagnosis for head and neck masses and is a reminder of the diverse etiologies for tumors in this region [4]. There was no suggestion of visceral disease on clinical presentation [4]. The patient denied gastric pain, decreased appetite, or weight loss [6], consistent with the finding of metastases in $80 \%$ of patients with gastric cancer at time of diagnosis [7]; for this reason, Suh et 
al. suggested to perform excisional biopsy of suspect lymph node to detect these tumors in early stage [8]. Metastasis of gastric cancer to the head and neck is less common [5], although VN (Troisier's sign) is well recognized [3].

The Virchow's node observed in visceral malignancy arises from tumor metastasis via the thoracic duct, so it very very rare observing a jump in the lymph nodes station during cancer metastasizing $[9,10]$. VN may compress or invade neurovascular structures, causing thoracic outlet syndrome, brachial plexopathy, phrenic neuropathy, or Horner's syndrome. Because of diverse sources of visceral malignancy, including gastrointestinal, hepatobiliary, and other origins, Virchow's node and its variants are important across surgical specialties. Diagnosis of neck masses is inherently challenging, and the presence of V level lymph node without Virchow's node is an atypical presentation that may obscure the source of the primary tumor. The most important point of this clinical case is the extreme rarity in identifying V lymph node without Virchow's node involvement [10], in fact, another case only has been reported in literature in 2008 by Takashima et al [11].

FUNDING : none.

FINANCIAL DISCLOSURE/CONFLICT OF INTEREST: The authors have no financial conflicts of interest relevant to this article to disclose.

ETHICAL APPROVAL AND CONSENT TO PARTECIPATE: the study was approved by the IRB of the hospital without releasing of an authorization number. The patient signed a written consent before being included in the study.

PATIENT CONSENT FOR PUBLICATION: the patient authorized to publish her data previous anonymization.

AVAILABILITY OF DATA AND MATERIALS: original data are available under request to the corresponding author.

AUTHORS' CONTRIBUTION: ADS, LdA, MB: study design and article writing; GL, PG, MP and TT collection of clinical data; GR and MM literature review, criticism and review of paper.

ACKNOWLEDGEMENT: not applicable

\section{REFERENCES}

1. Pynnonen MA, Gillespie MB, Roman B, et al. Clinical Practice Guideline: Evaluation of the Neck Mass in Adults. Otolaryngol Head Neck Surg. 2017;157: S1-S30.

2. Haynes J, Arnold KR, Aguirre-Oskins C et al. Evaluation of neck masses in adults. Am Fam Physician. 2015; 91:698-706.

3. Aghedo BO, Kasi A. Virchow Node. SourceStatPearls [Internet]. Treasure Island (FL): StatPearls Publishing; 2020.

4. Aguirre LE, Salcedo J, Zuquello R et al. Metastatic involvement of skeletal muscle from gastric adenocarcinoma. Oxf Med Case Reports. 2019; 2019: omz081.

5. Goto S, Takeda H, Sasahara Y et al. Metastasis of advanced gastric cancer to the extraocular muscle: a case report. J Med Case Rep. 2019; 13:107.

6. Sundriyal D, Kumar N, Dubey SK et al. Virchow's node. BMJ Case Rep. 2013; pii: bcr2013200749.

7. Karimi P, Islami F, Anandasabapathy S, Freedman ND, Kamangar F. Gastric cancer: descriptive epidemiology, risk factors, screening, and prevention. Cancer Epidemiol Biomarkers Prev . 2014;23(5):700713. doi: 10.1158/1055-9965.EPI-13-1057

8. Suh YS, Yang HK. Screening and Early Detection of Gastric Cancer: East Versus West. Surg Clin North Am . 2015;95(5):1053-1066. doi: 10.1016/j.suc.2015.05.012

9. Zdilla MJ, Aldawood AM, Plata A, Vos JA, Lambert HW. Troisier sign and Virchow node: the anatomy and pathology of pulmonary adenocarcinoma metastasis to a supraclavicular lymph node. Autops Case Rep . 2019;9(1): e2018053. Published 2019 Feb 25. doi:10.4322/acr.2018.053. 
10. López F, Rodrigo JP, Silver CE, et al. Cervical lymph node metastases from remote primary tumor sites. Head Neck . 2016;38 Suppl 1(Suppl 1):E2374-E2385. doi:10.1002/hed.24344

11. Takashima T, Nakata B, Hatama M, et al. Gastric cancer with cervical lymph node metastasis as the first presentation: report of a case. Int Surg. 2008; 93:295-299.

\section{FIGURE LEGENDS}

Figure 1: AB) MRI showing a metastatic lesion of the neck with histology from primary lesion. Coronal contrast-enhanced T1-weighted MRI slices show the enhancing lesion of the left posterolateral neck (asterisks). The lesion's location, encroaching on sternocleidomastoid musculature and prevertebral fascia, correlates with clinical symptoms of neck pain with lateral movement. CD) CT scan parasagittal and coronal view confirmed the presence of the mass (asterisk) and how it infiltrates surrounding structures. The round circle indicates norma lymph node.

Figure 2: Histology 200x magnification. A) Incisional biopsies of the left sternocleidomastoid muscle and superficial cervical fascia. Hematoxylin-eosin staining shows histological features suggestive of infiltrating gastric carcinoma (G2). B) Immune histochemistry with CKAE1/AE3 shows positive nuclear coloration indicative of cancer. C) Eosin and hematoxylin staining of gastric tissue from endoscopy shows classic histology of signet-ring cell carcinoma with characteristic signet-ring cell vacuoles (black arrow).

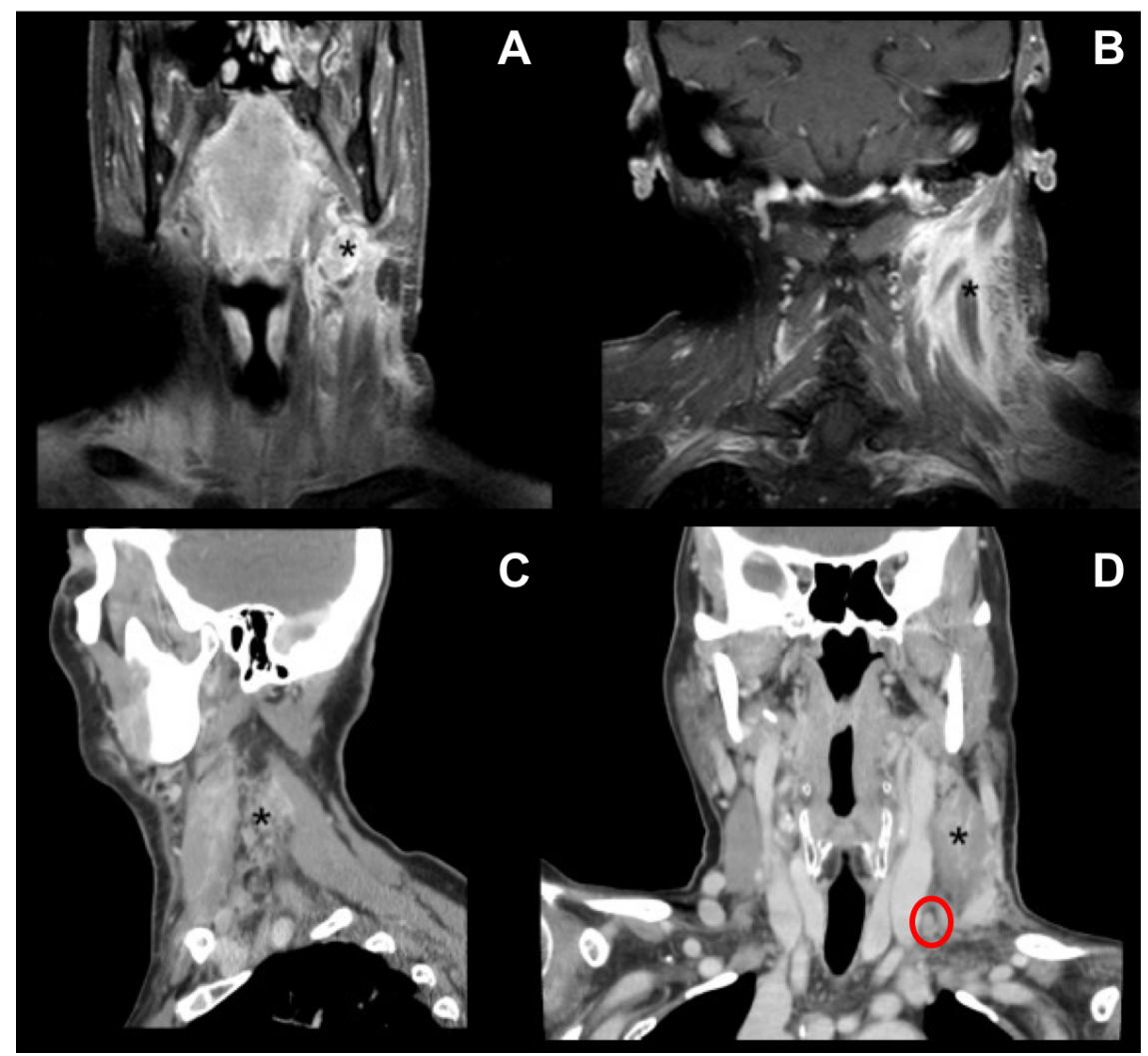




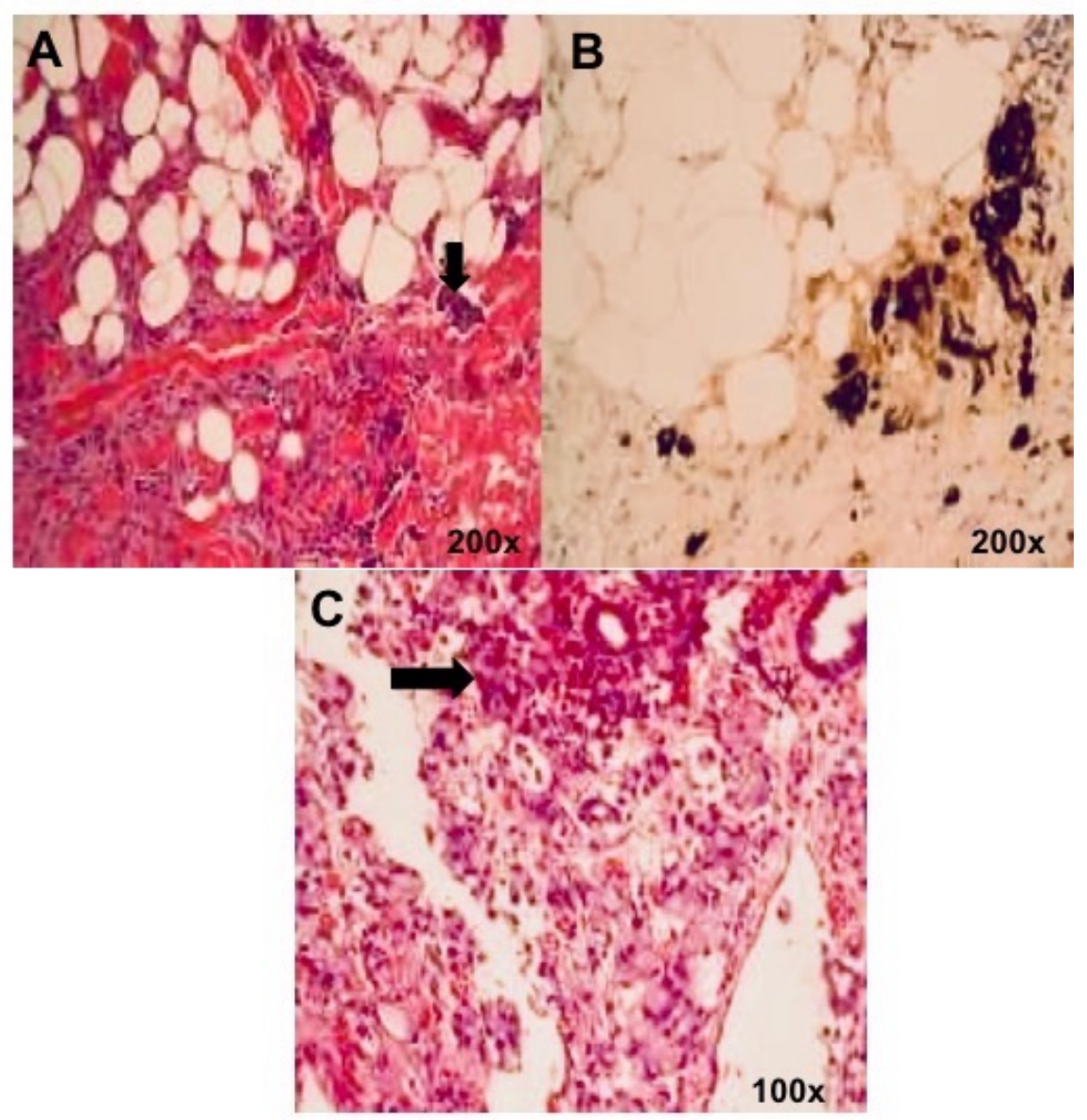

